\title{
Correction to: Alternative to Morse-Novikov theory for a closed 1-form. I
}

\section{Dan Burghelea ${ }^{1}$}

Published online: 24 January 2022

(c) Springer Nature Switzerland AG 2022

\section{Correction to: European Journal of Mathematics (2020) 6:713-750 https://doi.org/10.1007/s40879-019-00368-x}

1. The proof of Theorem 3.2 on p. 723 is incorrect. The statement is conjecturally true for all TC1-forms and true for a large class of TC1-forms; in particular for forms of degree of irrationality $\leqslant 1$ which are dense in the set of all TC1-forms, as well as for many other forms in any degree of irrationality class.

2. All statements including the proofs (except Theorem 3.2) are true as stated provided in the definition of "tame TC1-form" one adds the requirement that

$$
\lim _{t \rightarrow-\infty} H_{r}\left(\tilde{X}_{t}\right) \rightarrow H_{r}(\tilde{X})
$$

is injective. The requirement is (conjecturally) superfluous but this is not yet established in full generality.

3. In view of these comments a revised version of this paper will be posted on arXiv.

Publisher's Note Springer Nature remains neutral with regard to jurisdictional claims in published maps and institutional affiliations.

The original article can be found online at https://doi.org/10.1007/s40879-019-00368-x.

$凶$ Dan Burghelea

burghele@math.ohio-state.edu

1 Department of Mathematics, The Ohio State University, 231 W 18th Ave., MA 236, Columbus, $\mathrm{OH} 43210$, USA 